\title{
Dual-energy Computed Tomography for the Evaluation of Calcinosis in Systemic Sclerosis
}

VIVIEN HSU, MD, Associate Professor of Medicine, Department of Medicine, Rutgers-Robert Wood Johnson Medical School; MARK BRAMWIT, MD, Assistant Professor of Radiology, University of Radiology Group, Robert Wood Johnson University Hospital; NAOMI SCHLESINGER, MD, Professor of Medicine, Department of Medicine, Rutgers-Robert Wood Johnson Medical School, New Brunswick, New Jersey, USA. Address correspondence to Dr. V. Hsu, Adult Clinical Research Center, 51 French St., 3rd floor Acute Care Building, New Brunswick, New Jersey 08903, USA.

E-mail: hsuvm@rwjms.rutgers.edu. J Rheumatol 2015;42:345-6; doi:10.3899/jrheum.140773

Dual-energy computed tomography (DECT) imaging can be useful in the evaluation of scleroderma-related calcinosis (SSc calcinosis) of the hands.

We performed DECT scans of the hands of patients with symptomatic SSc calcinosis to better characterize the location and radiographic details of the calcinosis and
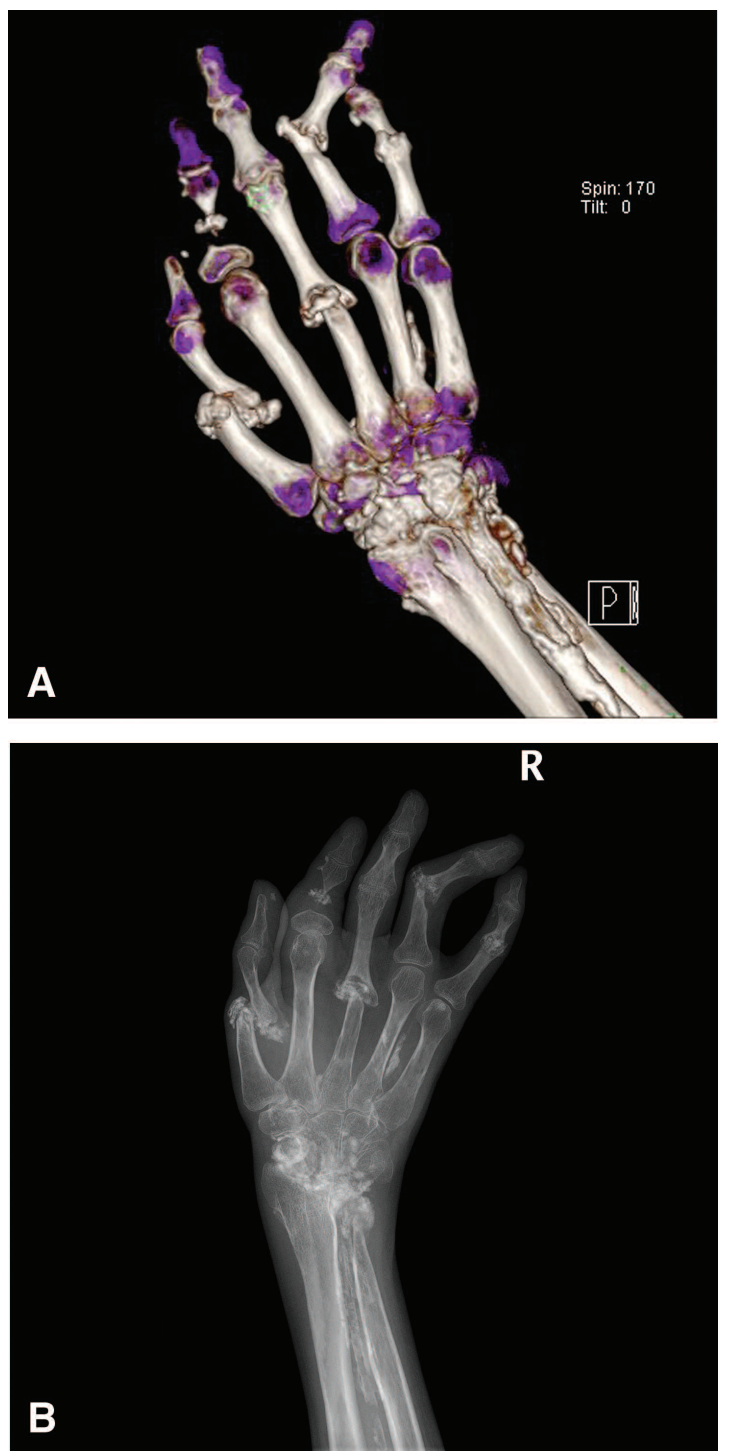

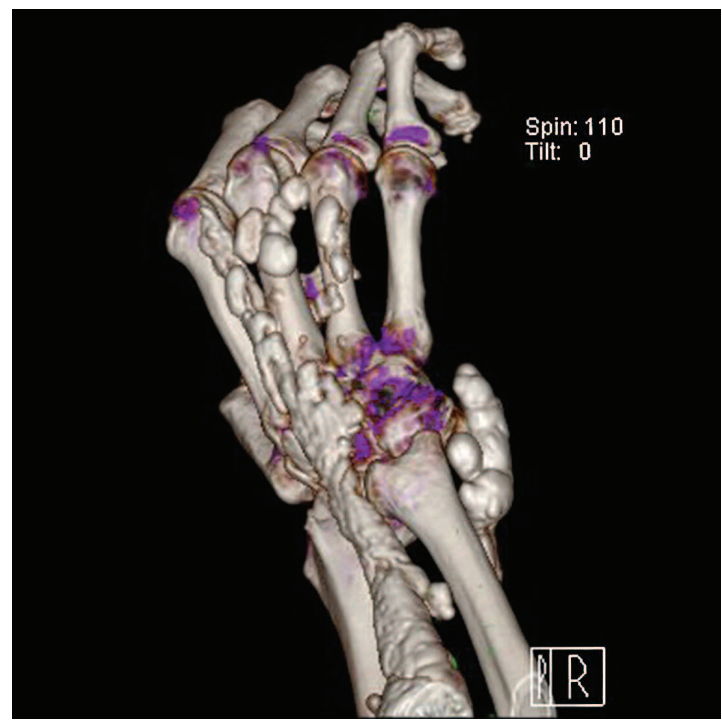

Figure 2. Severe acroosteolysis of all the digits of the right hand and flexion deformities of all the interphalangeal joints of the second through fifth fingers, of a woman with a 14-year history of diffuse systemic sclerosis. There is calcinosis within the soft tissues of all the fingertips, and severe calcinosis within the extensor and flexor muscles of the distal forearm and along the dorsal and volar aspects of the carpal bones. Dorsally, this extends from the forearm to the level of the distal metacarpal bones.

exclude any monosodium urate (MSU) crystal deposition. DECT is an advanced imaging modality useful for assessing soft tissue MSU deposition attributable to gout and visualization of bone and soft tissue structures such as ligaments and tendons $1,2,3$

We present here DECT images from 2 patients with SSc calcinosis. Figure 1 is the right hand and wrist of an African American woman with 10 years of diffuse SSc and seropos-

Figure 1. Two views of the right hand of a woman with 10 years of diffuse systemic sclerosis and seropositive polyarthritis. Erosive and degenerative changes are present at the first and third MCP, second DIP, and fourth and fifth PIP joints. The first MCP joint is dislocated. There are extensive soft tissue calcifications seen throughout the fingers and carpal bones with marked destruction and erosions at the radiocarpal joint. The scaphoid may be partially collapsed. The distal portion of the proximal second phalanx and the proximal portion of the middle second phalanx demonstrate severe tapering/penciling and are nearly completely resorbed. There are soft tissue calcifications surrounding the remaining waist of the proximal second phalanx. MCP: metacarpophalangeal; DIP: distal interphalangeal; PIP: proximal interphalangeal.

Personal non-commercial use only. The Journal of Rheumatology Copyright (c) 2015. All rights reserved. 
itive polyarthritis, whose calcinosis presented 2 years ago as painful wrist swelling. She failed surgical debulking and these painful deposits continue to drain from the wrist. The plain radiograph shows similar changes but in lesser detail, particularly in osteopenic areas where there are large deposits of SSc-calcinosis overlying the bone destruction.

Figure 2 is the right hand of a white woman with a 14-year history of diffuse SSc, whose calcinosis presented 8 years ago in both arms and hands. The hand is completely disabled from these enlarging and widespread deposits.

DECT imaging found calcinosis deposits most commonly in the subcutaneous fat pads of the fingertips, along tendon sheaths, within the carpal tunnel, and adjacent to muscle groups. No MSU crystal deposition has been identified. We propose that DECT imaging can be a useful imaging modality to assess SSc-calcinosis of the hands.

\section{REFERENCES}

1. Girish G, Melville DM, Kaeley GS, Brandon CJ, Goyal JR, Jacobson JA, et al. Imaging appearances in gout. Arthritis 2013;2013:673401.

2. Choi HK, Al-Arfaj AM, Eftekhari A, Munk PL, Shojania K, Reid $\mathrm{G}$, et al. Dual energy computed tomography in tophaceous gout. Ann Rheum Dis 2009;68:1609-12.

3. Deng K, Sun C, Liu C, Ma R. Initial experience with visualizing hand and foot tendons by dual-energy computed tomography. Clin Imaging 2009;33:384-9. 